Gangi, Lisa

[62]

\section{Gene expression in prostate cancer: microarray analysis of tumor specimens from African American and Caucasian patients}

Lisa Gangi ${ }^{1}$, Garrison Owens ${ }^{1}$, Walter Rayford ${ }^{2}$, Edison Liu ${ }^{1}$ $\&$ David Munroe ${ }^{1}$

${ }^{1}$ National Cancer Institute, National Institutes of Health, Bethesda, Maryland, USA

${ }^{2}$ Louisiana State University, Baton Rouge, Louisiana, USA

Adenocarcinoma of the prostate is the most frequently diagnosed cancer and the second leading cause of cancer deaths among American men. Prostate cancer has an even more devastating impact on the African American community: African American men have the highest incidence of prostate cancer in the world. At diagnosis, these patients are significantly younger in age and demonstrate a more advanced stage of the disease than Caucasian men. The mortality rate of prostate cancer is approximately $50 \%$ higher among African American men than among Caucasian men. Although there is mounting clinical evidence that the etiology of prostate cancer in African American men differs from that in Caucasian men, there is little understanding of the underlying molecular mechanisms. Complementary DNA microarrays allow the simultaneous assessment of thousands of genes in order to determine if there is a differential gene expression pattern that consistently segregates according to race and tumor grade. This microarray study compares total RNA samples extracted from histologically graded and Gleason-scored tumor prostectomy specimens from 20 African American and 10 Caucasian cancer patients.

Gavin, Mark

\section{Control of exit from $\mathrm{G} 1$ and $S$ phases of the cell cycle by histone deacetylase-Rb- hSWI/SNF and Rb-hSWI/SNF repressor complexes}

Mark Gavin, Steve Zhang, Anjali Dahiya, Robin Luo, Antonio Postigo, Duanduan Ma, William Harbour \& Douglas Dean

Division of Molecular Oncology, Washington University School of Medicine, St. Louis, Missouri, USA

The retinoblastoma protein $(\mathrm{Rb})$ has traditionally been associated with regulating the progression of cells through the G1 restriction checkpoint of the cell cycle. There is increasing evidence that $\mathrm{Rb}$ is also involved in controlling the progression of cells through $\mathrm{S}$ phase. We have evidence that $\mathrm{Rb}$ combines with a histone deacetylase and the ATP-dependent chromatin remodeler, hSWI/SNF, to form a complex that arrests cells in G1. After phosphorylation of Rb by cyclin D1/cdk4/6, the histone deacetylase dissociates from this complex, leaving the Rb-hSWI/SNF complex to regulate exit from $\mathrm{S}$ phase. We suggest that these complexes regulate cell cycle progression by controlling the order of expression of cyclins $\mathrm{E}$ and $\mathrm{A}$.
Giannella-Neto, Daniel

[64]

Identification of protein-tyrosine kinase
catalytic domain conserved sequences in
the ORF expressed sequence tags database

Daniel Giannella-Neto ${ }^{1}$, Maria Lúcia C. Correa ${ }^{1}$, Ana Mercedes S. Cavaleiro-Luna ${ }^{1}$, Maria Angela H.Z. Fortes ${ }^{1}$, Cassio N. Coimbral', Leonardo S. Oliveira'1, Sandro J. De Souza ${ }^{2} \&$ Andrew J.G. Simpson ${ }^{2}$

${ }^{1}$ Laboratory for Human Nutrition and Metabolic Diseases (LIM-25), Hospital das Clínicas, University of São Paulo School of Medicine, São Paulo, Brazil

${ }^{2}$ Ludwig Institute for Cancer Research, São Paulo, Brazil

Given the importance of protein-tyrosine kinases (PTKs) in the signaling pathways that lead to cell proliferation, it is not surprising that they have been implicated in the onset and proliferation of cancers, diabetic retinopathy, atherosclerosis and psoriasis. We have selected 328 conserved sequences grouped into PTK families comprising 13 amino acids that include the Asp-Phe-Gly motif of PTK catalytic domains. We have searched approximately 700,000 complementary DNAs representing partial expressed gene sequences biased toward the central coding regions (ORF expressed sequence tags [ORESTES]) of the resulting human transcripts of a variety of human tumors. A compilation of the searched sequences was obtained from Human Cancer Genome Project data available on line at http//:www.ludwig.org.br. We have identified 150 different translated ORESTES containing the Asp-Phe-Gly motif, representing 31 PTK families, mostly related to Eph/Elk/Eck orphan receptors (7/13 no-matched sequences in GeneBank), HGF (5/9 no-matched), cyclin-dependent kinases (CDKs) and close relatives (8/9 nomatched), DAG-activated, phospholipid-dependent PKs (2/9 no-matched), the PDGFR family (1/8 no-matched), DDR/TKT (1/7 no-matched), MEK/STE7 (0/7 no-matched), PAK/STE20 (0/6 no-matched), epidermal growth factor receptor (0/5 no-matched), SRC (3/5 no-matched), RAC(AKT) protein kinase (2/5 nomatched), and RAF (0/5 no-matched). Twelve other PTK families were also matched with fewer than than four ORESTES. Searching for PTK catalytic domains in the ORESTES database could provide a source for new sequences partially coding PTKs that could play a role in the progression of human cancer and reveal potential targets for PTK inhibitors.

Gieseg, Michael

[65]

\section{Expression profiling renal tumors as a first step in the evaluation of tumor models}

Michael Gieseg ${ }^{1}$, Mark Rubin $^{2}$, Theresa Cody ${ }^{1}$
$\&$ Eric Kaldjian

${ }^{1}$ Pfizer Global Research and Development, Ann Arbor, Michigan 48105, USA

${ }^{2}$ University of Michigan, Ann Arbor, Michigan 48105, USA

Tumor models are poorly predictive of the efficacy of anticancer drugs on human neoplasms. Expression profiling technology presents a rational approach to identifying and unraveling the complex environmental and genetic differences that exist between models and human disease. As a first step in identifying these differences, we are building an expression database of renal cell carcinomas, comparing messenger RNA levels of primary tumors with those of normal kidney cortex from the same patient removed during surgical resection. Four tumor/normal kidney pairs were analyzed using the Affymetrix HuGeneFL array to determine if this approach could functionally separate subtypes of renal cell carcinoma. Two carcinomas were a clear-cell type and two were chromophobe carcinomas. Analysis of the mRNA expression results revealed that the normal samples clustered separate- 


\section{Abstracts: Session I}

ly from the tumors. Within the tumor group, the clear-cell and chromophobe carcinomas demonstrated markedly distinct patterns of gene expression. To assess the functional differences between the two types, we selected genes using a twofold change threshold in expression common to both samples of each tumor type and assigned functional classification to each gene where possible. Gene function was divided into 16 different cellular categories using the Saccharomyces cerevisiae functional categories of the Munich Information Center for Protein Sequences as a guide. Graphical representations of the numbers of genes from each category indicated that the clear-cell tumors might have different cellular functions than the chromophobes. We will use this database in future work to assess the differences between animal xenografts and human renal tumors.

Gillanders, E. [66]

\section{Use of experimentally constructed haplotypes in gene mapping studies of hereditary cancers}

E. Gillanders ${ }^{1}$, J.A. Douglas², S.B. Gruber ${ }^{3}$, H. Yan ${ }^{4}$, B. Vogelstein ${ }^{4}$, R. Sood ${ }^{1}$, J. Carpten ${ }^{1}$, T. Dennis ${ }^{1}$, M. Boehnke², J.M. Trent

${ }^{1}$ Cancer Genetics Branch, NHGRI, Bethesda, Maryland, USA

${ }^{2}$ Depts. of Biostatistics, Epidemiology, and ${ }^{3}$ Internal Medicine, Univ Michigan, Ann Arbor, USA

${ }^{4}$ Howard Hughes Medical Institute and The Johns Hopkins Oncology Center, Baltimore, Maryland, USA

The technique of conversion provides several advantages for gene mapping projects of complex diseases such as cancer. The approach takes advantage of selective retention of a subset of human chromosomes within somatic cell hybrids, isolating single copies of all desired human chromosome pairs. This strategy functions both to simplify mutation detection, as well as allow unambiguous phase information to be determined. Constructing haplotypes on the basis of conventional genotype and pedigree data is challenging, particularly for late age of onset diseases such as cancer.

Identification of which homolog is retained in a particular hybrid is determined by conventional genotyping of a few markers per chromosome. These 'haploid' hybrids can then be used to increase the sensitivity of traditional mutation analysis as the disease causing chromosome will not be accompanied by the normal wild-type allele. Additionally, haplotypes maybe determined simply by genotyping each haploid hybrid. We have typed DNA from 100 hybrids to examine chromosomal retention patterns, test the feasibility of conversion for whole genome analysis, and evaluate assumptions regarding appropriate experimental design.

We have investigated the theoretical efficiency of using haplotypes compared to conventional genotypes in linkage and linkage disequilibrium studies. In the linkage disequilibrium setting, we determined the Fisher information (with respect to haplotype frequency) provided by unrelated individuals. For two-SNP haplotypes, conversion provides $5-45 \%$ more information per subject than standard genotyping, depending on true haplotype frequencies; for five-SNP haplotypes, improvement ranges $20-92 \%$. As Fisher information is inversely related to sample size, conversion requires up to 12.5 times fewer subjects than standard genotyping to obtain the same information. We currently are completing similar calculations in the linkage analysis setting. The extra cost associated with conversion includes hybrid construction and characterization, and duplicate genotyping. However, the increased information per subject reduces overall recruitment and phenotyping costs, which tend to dominate those for genotyping. The efficiency of using conversion to construct haplotypes is likely to increase even further as automated methods of genotyping continue to improve.
Gokgoz, Nalan

[67]

\section{Analysis of gene expression patterns in breast cancer by microarray technology}

Nalan Gokgoz ${ }^{1}$, Xiang Sun ${ }^{2}$, Shelly Bull ${ }^{1}$, Jim Woodgett ${ }^{2} \&$ Irene Andrulis ${ }^{1}$

${ }^{1}$ Samuel Lunenfeld Research Institute, Mount Sinai Hospital, 600 University Avenue, Toronto, Ontario M5G 1X5, Canada

${ }^{2}$ Ontario Cancer Institute, Princess Margaret Hospital, 610 University Avenue, Toronto, Ontario M5G 2M9, Canada

Although alterations in several specific genes have been implicated in breast cancer progression, greater understanding of the molecular basis of the disease may result from the evaluation of global gene expression patterns using microarray technology. To identify patterns of gene expression of prognostic importance in axillary node-negative breast cancer, we are studying a large cohort of patients with this type of cancer. Before using the limited amount of RNA from these specimens, we performed pilot studies designed to evaluate the feasibility of applying the technology on a larger scale. We studied gene expression in four different breast cancer cell lines (T47D, MDA231, SKBR3 and BT474) using complementary DNA microarrays containing 1,700 and 19,000 sequence-verified human cDNAs produced by the microarray facility at the Ontario Cancer Institute, Toronto (http://www.oci.utoronto.ca/services/microarray). Each hybridization compared Cy5-labeled complementary DNA from one of the cell lines with Cy3-labeled cDNA from a reference sample (MCF12A, a normal breast cell line). We also performed reciprocal labeling with subsequent hybridization to demonstrate the consistency and reproducibilty of the technology. In addition, different amounts of RNA $(50 \mu \mathrm{g}, 25 \mu \mathrm{g}$ and $10 \mu \mathrm{g})$ from the same cell lines were labeled to determine the sensitivity of the system. We have been able to devise a system that can now be applied to primary breast tumors. After expression analysis, we will use biostatistical modeling to detect clusters of genes that are coordinately expressed, repressed or both. These clusters are likely to represent common pathways of genes involved in breast carcinogenesis.

Golubic, Mladen

$[68]$

\section{Genetic basis of radiation response in glioblastoma multiforme}

Mladen Golubic, Olga Chernova, LesleyAnn Hawthorn, Sofia Chernova, James Evans, Kathy Signorelli, John Cowell \& Gene Barnett

\section{Cleveland Clinic Foundation, 9500 Euclid Avenue, Cleveland, Ohio 44195, USA}

Glioblastoma multiforme (GBM) is an almost uniformly fatal brain tumor; patients have a median survival time of less than one year despite aggressive treatments, including surgery, radiation and chemotherapy. Despite the clear benefits of radiation therapy in prolonging the survival of some patients with GBM, only about one-third of them demonstrate an objective radiographic response. To pinpoint the genetic difference between GBMs that responded well to radiation treatment and those that did not, we analyzed the gene expression profiles of two nonresponding (NR) and two responding (R) tumors using Affymetrix Hu6800 oligonucleotide arrays. Comparison between two paired tumor samples ( $\mathrm{R}$ versus NR) revealed that 423 and 236 genes were differentially expressed (sort score 0.5 ). Of those, 33 genes showed consistently increased or decreased expression in both $\mathrm{R}$ tumors compared with NR tumors. These differentially expressed genes are known to regulate cell motility, cellular responses to DNA damage, cell cycle, angiogenesis and apoptosis. For example, decreased expression of genes known to stimulate tumor cell motility and increased expression of genes that inhibit cell migration was observed in $\mathrm{R}$ tumors. We also determined the gene expression of 